\title{
Observation of Single Atoms in Liquid and Liquid Inhomogeneous Structures
}

\author{
Tomohiro Miyata ${ }^{1}$ and Teruyasu Mizoguchi ${ }^{1}$
}

1. Institute of Industrial Science, The University of Tokyo, Tokyo, Japan.

Liquid supports our life and industrial activities as transport carriers and reaction media. Thus, investigation of liquid behavior and the reaction mechanism is crucial for both basic research and application. Although liquid is often considered to have a random homogeneous structure, it actually forms inhomogeneous structures in molecular-scale [1-3], and these structures are known to dominate the liquid properties. In addition, macroscopic substance diffusions and chemical reactions are derived from the accumulation of each molecular behavior in a local area. Therefore, atomic/molecular scale investigation of liquid is indispensable for further development of liquid applications. However, because the existing liquid analysis methods, such as x-ray diffraction and optical spectroscopies, are not good for local area investigation, more advanced ones with high spatial resolution has been desired. Here, we have adopted aberration-corrected scanning transmission electron microscopy (STEM) with atomic resolution as the liquid analysis method. In this study, we addressed observations of single atoms in liquid and nano inhomogeneous structures that molecules form.

In general, liquid observations using TEM/STEM have been conducted by the liquid-cell method, which sandwiches liquid between two solid films, so that the liquid does not evaporate under vacuum $[4,5]$. However, the spatial resolution is deteriorated owing to the total sample thickness, and this makes it difficult to observe single atoms and liquid nano structures using this method. That is, in order to keep the atomic resolution, a thin liquid sample without the solid films should be prepared. Therefore, ionic liquid, having almost no vapor pressure, was selected as the observed liquid. The ionic liquid was dropped on a holey solid film to form liquid films in the holes by surface tension. Figure 1 shows illustrates the fabrication process. The thickness of the liquid film was measured at about $10 \sim 50 \mathrm{~nm}$ $[6,7]$. Because this value is much less than the mean free path length, this liquid sample can provide the observation with the original atomic resolution.

Next, in order to achieve the observation of single atoms in liquid, we added gold complex ions $\left(\mathrm{AuCl}_{4}^{-}\right)$ into an ionic liquid $\mathrm{C}_{8}$ mim-Cl and observed with high angle annular dark field STEM (HAADF-STEM). Since the HAADF-STEM intensity is approximately proportional to the square of atomic number Z, the gold atoms $(Z=79)$ in $\mathrm{C}_{8}$ mim- $\mathrm{Cl}$, composed of light elements $(Z \leqq 17)$, would be observed very bright. Figure 2(a) shows the HAADF-STEM image of $\mathrm{C}_{8} \mathrm{mim}-\mathrm{Cl}$ with gold atoms. In this image, many bright spots considered as the gold atoms are observed. The histogram of the spot size is shown in Fig.2(b). The peak of the fitted gauss function appears at about $0.0085 \mathrm{~nm}^{2}$, converted into the circle diameter of $0.10 \mathrm{~nm}$. This diameter is in good agreement with the electron probe diameter of about $0.08 \mathrm{~nm}$, concluding that almost all of the observed bright spots are according to single gold atoms. In addition, we analyzed the behavior of the atoms by obtaining the continuous images. Nano inhomogeneous structures in ionic liquid $\mathrm{C}_{8} \mathrm{mim}-\mathrm{Cl}$ was also investigated from the coordination of the gold atoms. We will report the detailed results in our presentation with movies of the atomic movement.

\section{References:}

[1] C. Huang et al, PNAS 106 (2009) p. 15214. 
[2] O. Yamamuro et al, J. Chem. Phys. 135 (2011) p. 054508.

[3] A. Triolo et al, J. Phys. Chem. B 111 (2007) p. 4641.

[4] N. de Jonge and F. Ross, Nature Nanotech. 11 (2011) p. 695.

[5] M. Nielsen, S. Aloni and J. De Yoreo, Science 345 (2014) p. 1158.

[6] T. Miyata and T. Mizoguchi, Microscopy 63 (2014) p. 377.

[7] T. Miyata and T. Mizoguchi, submitted.

[8] This study was supported by NIMS microstructural characterization platform (NMCP) as a program of "Nanotechnology Platform" of MEXT, Japan. The authors also supported by funding from Mitsubishi Science Foundation (27143) and Grants-in-Aid for Scientific Research from MEXT (25106003, 26630302, 26249092). T. Miyata acknowledges a Grant-in-Aid from the JSPS Research Fellows (15J11146). The authors are grateful to Mr. F Uesugi in NMCP for supporting and discussing this work.

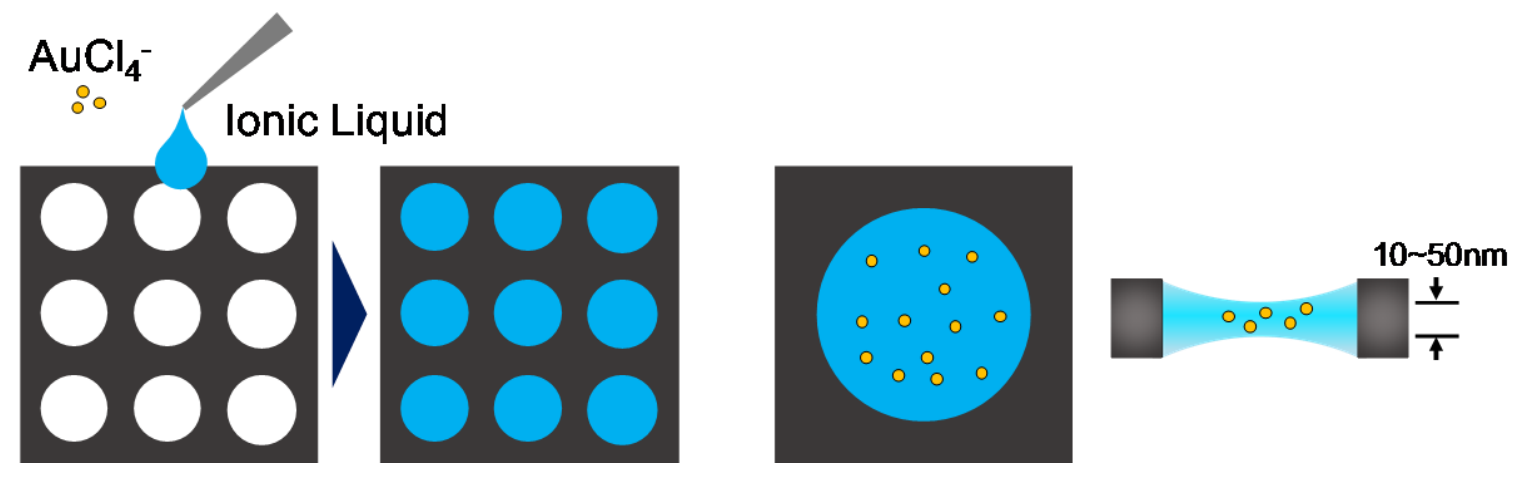

Figure 1. Illustration of the fabrication method of the liquid sample.
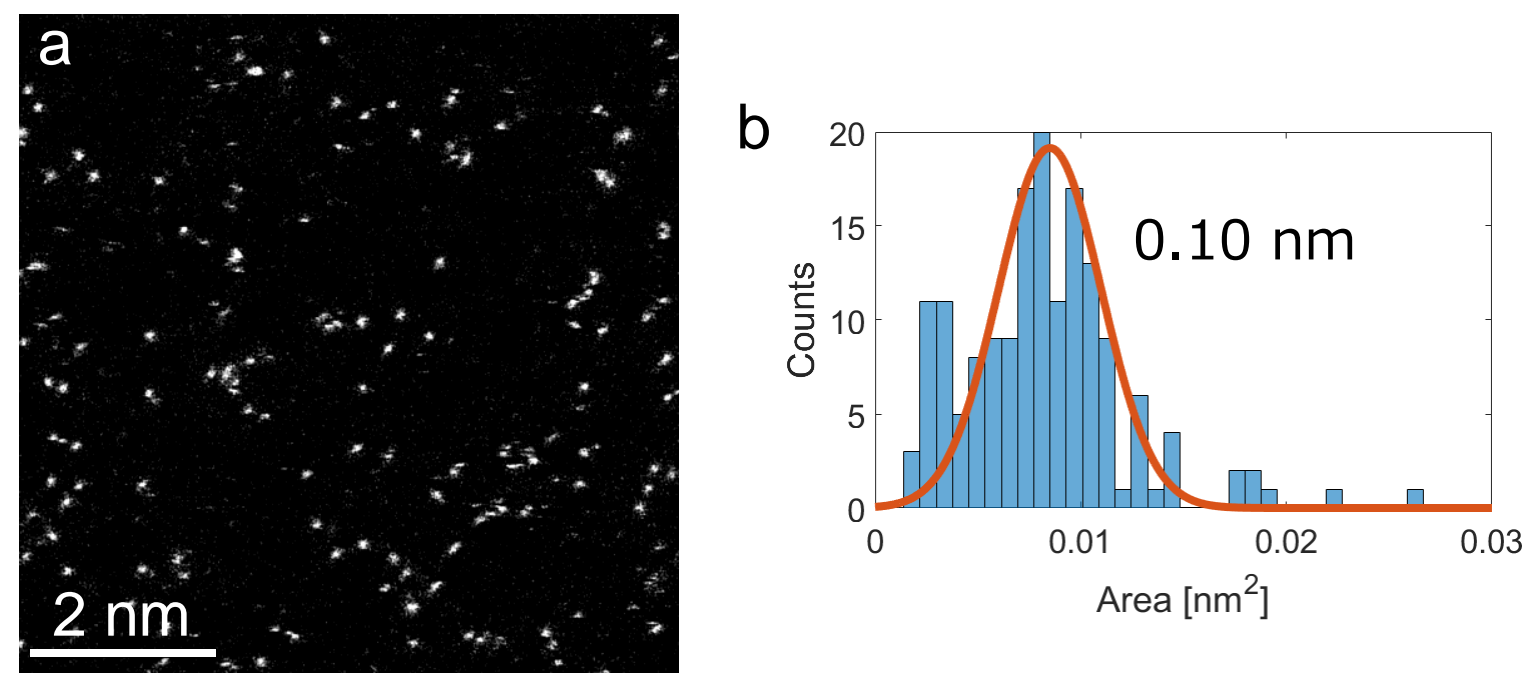

Figure 2. (a) HAADF-STEM image of $\mathrm{C}_{8}$ mim-Cl with $0.1 \mathrm{M} \mathrm{AuCl}_{4}^{-}$, (b) histogram of the bright spot size in (a). 